\title{
Controlling Electric Vehicles in Quasi-Real-Time
}

\author{
F. J. Soares, Student Member, IEEE \\ Power System Unit \\ INESC TEC (formerly INESC Porto) \\ Porto, Portugal \\ fsoares@inescporto.pt
}

\author{
J. A. Peças Lopes, Senior Member, IEEE \\ Power System Unit \\ INESC TEC (formerly INESC Porto) / FEUP \\ Porto, Portugal \\ jpl@fe.up.pt
}

\begin{abstract}
This work presents a methodology to manage Electric Vehicles (EV) charging in quasi-real-time, considering the participation of EV aggregators in electricity markets and the technical restrictions of the electricity grid components, controlled through the distribution system operator. Two methodologies are presented to manage EV charging, one to be used by the EV aggregators and the other by the Distribution System Operators (DSO). The methodology developed for the aggregator has as main objective minimizing the deviation between the energy bought in the market and the energy consumed by EV. The methodology developed for the DSO allows it to manage the grid and solve operational problems that may appear by controlling EV charging. A method to generate a synthetic EV data set is used in this work, which provides information about the EV movement, periods when EV are parked, as well as their energy requirements. This data set is used afterwards to assess the performance of the algorithms developed to manage the $\mathrm{EV}$ charging in quasi-real-time.
\end{abstract}

Index Terms-Aggregators, Electric Vehicles, Electricity Markets, Load Management, Quasi-Real-Time Management.

\section{INTRODUCTION}

The expected deployment of Electric Vehicles (EV) will considerably affect the way distribution grids will be managed and operated in the future. The extra amount of power they will demand from the grid will oblige Distribution System Operators (DSO) to understand the impacts resulting from EV connection to distribution grids. Several approaches to this problem have been presented lately in the literature.

In $[1,2]$, the authors analyzed the changes in the load diagrams of distribution networks for increasing penetration of EV. Lopes et al., in [3], also studied the impacts of EV on distribution grids. The novelty introduced by these authors was the evaluation of the EV charging impact on the grid technical constraints, like voltages and branches' congestion levels. Clement et al., in [4], analyzed the Plug-in Hybrid EV (PHEV) impacts on distribution grids' power losses and voltage deviations. Although the methodologies proposed in papers [1-4] revealed to be interesting approaches to evaluate EV impacts, they do not provide an adequate method to determine the optimal EV charging schedules in quasi-realtime. It should be noted that for the purpose of this work, the term "quasi-real-time" is used in the sense of monitoring the grid and managing EV in a short period of time, like 5 to 10 minutes (or less, depending of the effectiveness of the communication infrastructure).

Several other works have been developed with the main purpose of determining the optimal (or near optimal) EV charging schedules, [5-9]. However, some of these approaches are rather complex and time consuming, being impractical for online applications.

This paper presents an innovative approach that uses a holistic methodology to manage the EV charging in distribution grids in quasi-real-time, taking into account the concerns of all the players involved in the process: the grids' technical restrictions (DSO concern), the periods during which EV are parked (aggregators' concern), the EV owners' energy requests (EV owners' concern) and the operational requirements of electricity markets. The development of this work involved the creation of two expeditious methodologies to be used by aggregators and DSO to manage the EV charging in quasi-real-time, which allow, respectively: minimizing the aggregators' penalties for the deviations between the energy they bought in the markets and the energy sold to EV owners (imbalance settlement), thus contributing to increase the aggregators' profit; and solving technical problems related with voltages outside the operational ranges and branches' overloading that might appear in the grid. In order to assess the performance of these methodologies, a synthetic EV data set was used, which was created with an algorithm that uses a Markov chain to simulate the EV movement, as well as their power requirements.

\section{EV INTEGRATION FRAMEWORK FOR ISLAND POWER SYSTEMS}

Moving from a "fit-and-forget" policy to an active EV management context implies the creation of a suitable technical/commercial framework capable of dealing with the technical aspects of electricity grids and the markets operation.

\section{A. Control Structure to Manage EV in Quasi-Real-Time}

Under this new framework, when operating the grid in normal conditions, EV will be managed and controlled by a new entity - the aggregator - whose main functionality will be grouping EV, according to their owners' willingness, to exploit business opportunities in the markets [5]. If EV would 
enter the market individually their visibility would be small and due to their stochastic behavior their participation in the market would be impossible. Yet, if an aggregator exists, then the services potentially provided by EV would be more significant and the confidence on its availability much higher.

Nevertheless, even considering the EV aggregators' activities, a still high degree of uncertainty will exist related to when and where EV will charge. Due to these uncertainties and assuming that grids will evolve towards a decentralized generation paradigm, the existence of a grid monitoring structure, such as the one developed for micro-grids and multimicro-grids, will be required. This structure will be controlled by the DSO and should be capable of acting over EV charging in abnormal operating conditions, i.e. when the grid is being operated near its technical limits, or in emergency operating modes, e.g. islanded operation. This system should follow a hierarchical structure, from a central Distribution Management System (DMS) down to specific EV controllers [5].

It is important to stress that the aggregator should always take into account the EV owners' requests, which should provide information about the energy required and connection period via, for instance, the smart metering infrastructure [5]. The aggregator should have a hierarchical structure similar to the grid control system used by the DSO, as described in [5], to be capable of communicating and managing the EV charging in quasi-real-time. Both technical and market layers will require an advanced communication infrastructure to enable information exchange between all the involved players.

\section{B. Charging Levels Considered}

There are several types of EV charging solutions being currently adopted, [10], which involve distinct power levels: Level 1 - Around $3 \mathrm{~kW}$ charging power that can be obtained through common domestic outlets; Level $2-10-20 \mathrm{~kW}$ that can only be obtained through dedicated charging outlet and wiring; and Level 3 - More than $40 \mathrm{~kW}$ that can only be obtained through dedicated charging outlet and wiring and using a dedicated off-board charger for DC fast charging. The charging type commonly classified as slow refers to level 1 , while the fast charging refers to level 3 . Level 2 is an intermediary charging level. All the three levels were considered in this work, being assumed that slow charging corresponds to level $1-\mathrm{LV}$ connections, while fast charging includes level 2 and 3 - Medium Voltage (MV) connections.

\section{Charging Schemes Considered}

Depending on the type of application, EV controllability may vary and, therefore, several control schemes may be adopted. In the solutions involving fast charging (level 2 or 3), a full charge might take less than $1 \mathrm{~h} \mathrm{[10].} \mathrm{Due} \mathrm{to} \mathrm{the} \mathrm{urgent}$ needs from the user of these types of services, especially level 3 clients, no controllability is envisaged. On the other hand, depending on the EV battery State-of-Charge (SOC) and capacity, full charge solutions involving level 1 might take up to $12 \mathrm{~h} \mathrm{[10]}$. Within this charging alternative, it is assumed that EV owners can choose between a set of three charging options: two passive or non-controlled (dumb charging and multiple tariff) and one active or controlled (smart charging).
In the dumb charging, the EV owners are free to connect and charge their EV when they want and the charging starts automatically after the EV being plugged-in [5]. As in the previous approach, the dual tariff policy assumes that EV owners are free to charge their vehicles. Yet, electricity cost is assumed not to be constant during the day, existing some periods when its cost is lower [5], which may lead EV drivers to prefer charging during these periods. The smart charging envisions an active management system where there exists a hierarchical control structure headed by the SO, as referred in section II A, which is used to control the EV charging rates.

\section{QUASI-REAL-TIME MANAGEMENT OF EV CHARGING}

\section{A. Aggregators' Management}

The main objective of the proposed methodology is to define which smart charging adherents should charge at each time step, in order to minimize the deviations between the energy bought in the market by the aggregators and the energy consumed by EV. It should be stressed that it was assumed that the power charging rate for level 1, for smart charging adherents, could be controlled between 0 and $3 \mathrm{~kW}$. To achieve the intended objective, it is required to find a set of $n$ load values, being $\mathrm{n}$ the number of smart charging adherents, which can be defined as optimal in the sense that they allow minimizing the deviations referred above. This problem can be formulated as an optimization problem, as shown next.

$\min \mid E B A_{t}-$ TIEV $_{t}-\sum_{i=1}^{n} F E V L_{t}^{i} \mid$

subject to

$$
0 \leq S O C R_{t d}^{i}-S O C_{t}^{i} \leq \frac{\left(F E V L_{t}^{i}+(t d-(t+1)) \times 3\right) \times \frac{1}{2} \times E V_{c e}}{E V_{i}^{b c}} \times 100
$$

$0 \leq F E V L_{t}^{i} \leq 3$

$0 \leq \operatorname{SOCR}_{t d}^{i} \leq 100$

$0 \leq S O C_{t}^{i} \leq 100$

$t+1 \leq t d$

where:

- $\quad i$-represents the "flexible $\mathrm{EV}^{1}$ " index;

- $t$-represents the time index;

- $\quad n-$ is the nr. of "flexible EV" under the aggregator control;

- $E B A_{t}$ - represents the average power during $1 / 2 \mathrm{~h}$, in $\mathrm{kW}$, related with the energy bought in the day-ahead market by the aggregator for time period between $t$ and $t+1$; $E B A_{t}(k W)=$ energy bought $t_{t \rightarrow t+1}(k W h) / 1 / 2 h$;

- $\quad T I E V L_{t}$ - is the "inflexible $\mathrm{EV}^{2}$ " load, in $\mathrm{kW}$, in time step $t$;

- $\quad F E V L_{t}^{i}$ - is the power absorbed by "flexible EV" $i$, in $\mathrm{kW}$, in time step $t$; the $n F E V L_{t}^{i}$ are the decision variables of the optimization problem; they can assume continuous values in the interval $[0,3]$;

- $t d$ - represents the time step at which a given "flexible EV" disconnects from the grid;

- $\quad S O C_{t}^{i}$ - are the EV i battery SOC, in percentage, in time step t;

- $S O C R_{t d}^{i}$ - represents the battery SOC required by the owner of EV $\mathrm{i}$, in percentage, in time step td;

- $E V_{i}^{b c}$ - represents the battery capacity, in $\mathrm{kWh}$, of EV i;

- $E V_{c e}$-represents the efficiency of the EV charging process.

Equation (2) is used to assure that the EV battery SOC, required by the $\mathrm{EV}$ owners at the moment of disconnection, is possible to attain when considering a maximum charging rate of $3 \mathrm{~kW}$. Equation (3) assures that only charging rates

\footnotetext{
1 "Flexible EV" are the EV whose owners adhered to the smart charging.

2 "Inflexible EV" are the EV whose owners adhered to the dumb charging or multiple tariff schemes.
} 
between $[0,3] \mathrm{kW}$ will be attributed to "flexible EV", as it was assumed that a "flexible EV" is a smart charging adherent that charges either in a residential or industrial area at level 1 . Equations (4) and (5) are used to guarantee that the required battery SOC and battery SOC in the time step $t$ are always within the interval $[0,100] \%$. Equation (6) assures that the time of disconnection always takes place after time step $t+1$.

The objective of this optimization problem is then to minimize the sum of the absolute value of the deviations ${ }^{3}$. It is a linear optimization problem, which is suitable for quasi-realtime applications since it is very fast to solve and does not require any type of forecasted data. It is only needed to know, for the current time step (t), the energy bought by the aggregators, the power consumed by the "inflexible EV", the moment of disconnection of the "flexible EV" that are plugged-in and the energy required by their owners during the connection period. At this stage grid restrictions are not limiting EV charging, since the problem is being dealt only taking into account the market operation.

\section{B. DSO Management}

After defining which "flexible EV" should charge and with which charging rate at each time step, the grid operating conditions should be analyzed to detect eventual technical problems that may appear. If operational restrictions are violated, the DSO needs to define the amount of load that is required to decrease to bring voltages and branches' ratings again to the allowable limits and to define which of the "flexible EV" should decrease their charging rates in order to attain the desired load reduction.

A procedure to tackle these problems was developed, which is capable of tackling simultaneously multiple low voltage and lines overloading problems, whether these problems occur in separate or in the same feeder of a given network. Despite only providing near-optimal results, it allows rapidly identifying solutions to solve technical problems in the network (by changing EV load) with very satisfactory results. This approach is based in a heuristic with two stages:

1) In the first stage all the load data is gathered and, having knowledge of the network technical characteristics and topology, a power flow is run to evaluate its operating conditions. Then, a list of problematic buses is identified and these buses are sequentially analyzed. A given bus is flagged as problematic if it has a voltage value below $V^{\text {min }}$ or if it is located in the upstream end of a branch with a rating above $S^{\max }$. For each problematic bus, the feeder that contains the bus under analysis is selected and the amount of load that is required to decrease in each of the feeder's buses is calculated. This calculation is performed iteratively, by decreasing in steps of a fixed value, in this case assumed to be $10 \%$, the existing EV load in each of the feeder's buses. Yet, it should be noted that a different load step decrease can be adopted.

2) In the second stage, the "flexible EV" that should reduce their charging rates are selected, in order to decrease

\footnotetext{
${ }^{3}$ There are two types of deviations: positive and negative. In this work, it is assumed that positive deviations are referred to the situations where the energy bough by the aggregators is higher than the EV consumption, whereas negative deviations are referred to the opposite situation.
}

the amount of power calculated in the first stage. As this methodology was developed for MV and LV networks, considering three-phase balanced operation, the loads resulting from the EV batteries charging were modeled as three-phase balanced loads. Thus, all the "flexible EV" charging downstream a feeder that contains problematic buses are eligible to reduce their charging rates. Yet, only "flexible EV" that are capable of effectively contributing to solve the grid problems identified before are selected (this depends of their location in the grid).

It should be noted that in a first phase this heuristic process only reduces the charging rates of "flexible EV". Yet, when low voltage and lines overloading problems are so severe that the emergency operating state is triggered, this heuristic reduces the charging rates of all the EV located in the problematic areas of the grid, disregarding if they are "flexible EV" or "inflexible EV.

For MV network studies, the EV charging at level 1 are assumed to be connected to one of the LV grids that are downstream the MV grid. Yet, as in this simulation the MV grids were modeled until the MV/LV substation, the loads of the EV that are connected to a given LV grid are grouped and represented as a single load in the respective MV bus of the substation. When charging at levels 2 or 3, EV are assumed to be directly connected to the MV grid and thus their load will be allocated to the respective MV bus.

It should be mentioned that the two problems referred above, low voltage and lines overloading, could be solved using an Optimal Power Flow (OPF)-like method for distribution networks. However, as the resolution of this type of problems is usually very time-consuming, [11], the expeditious approach presented in this section was chosen over the OPF-like option since the latter is rather impractical for online applications, [12].

\section{Generation of the Synthetic EV Data Set}

The first step to generate the synthetic EV data set was to individually characterize all the EV assumed to be enclosed in a MV network used as case study (further details about the case study are provided in section V). After, it was simulated the movement of the EV fleet during one week, according to common traffic patterns (data from a region in the north of Portugal [13] was used). Having the EV movement defined, their power requirements were computed and the data obtained was used in the case study of section $\mathrm{V}$.

\section{A. Characterization of the EV Fleet}

Each EV was initially characterized in terms of battery capacity, charging power, energy consumption and battery SOC in the beginning of the simulation $(t=0)$. These values were defined according to truncated Gaussian probability density functions, whose average, standard deviation, maximum and minimum values are presented in Table I. The maximum and minimum values allowed of the referred functions were introduced in order to avoid having unrealistic values for these variables when making the draw for each EV. While the initial battery SOC values were assumed for the purpose of this work, the remaining values were gathered from the information made available by 42 different EV 
manufacturers. It was assumed that the global efficiency of the overall charging process was $90 \%$.

TABLE I. TRUNCATED GAUSSIAN DISTRIBUTIONS FOR EV CHARACTERIZATION

\begin{tabular}{ccccc}
\hline \hline & Average & Std. dev. & Max. & Min. \\
\hline Battery capacity $(\mathrm{kWh})$ & 24.73 & 17.19 & 85.00 & 5.00 \\
Slow charging power $(\mathrm{kW})$ & 3.54 & 1.48 & 10.00 & 2.00 \\
Energy consumption $(\mathrm{kWh} / \mathrm{km})$ & 0.18 & 0.12 & 0.85 & 0.09 \\
Initial battery SOC $(\%)$ & 75.00 & 25.00 & 95.00 & 25.00 \\
\hline \hline
\end{tabular}

A driver behavior was also assigned initially to each EV. The possible behaviors considered in this paper were obtained from a survey made within the framework of the MERGE project [10]. The results revealed that there are three major types of behaviors regarding EV charging: EV charge at the end of the day $(57 \%)$, EV charge only when it needs $(23 \%)$ and EV charge whenever possible (20\%). For the drivers who charge their EV only when it needs, it was assumed that the battery SOC that triggers the need for charging was $40 \%$.

\section{B. Simulation of the EV Movement}

The movement of the EV during the period of a week was simulated using a discrete-state, discrete-time Markov chain, as described in [14], to define the states of all the EV for each time step (with 30 minutes duration). It was assumed that, at every unit of time, each EV can be in one of the following states: in movement or parked in residential / commercial / industrial area. After defining the EV states for each time instant, a network bus location was attributed to parked EV, according to a probability distribution proportional to the load installed in each bus. For the EV in movement, a procedure was developed to account their energy consumption and the respective reduction in the battery SOC, as defined in [14]. At each time instant, the battery SOC is updated according to the energy spent travelling or absorbed from the grid. It was assumed that EV parked in residential and industrial areas charge at $3 \mathrm{~kW}$ (level 1), in commercial areas at $12 \mathrm{~kW}$ (level 2) and in fast charging stations at $40 \mathrm{~kW}$ (level 3). When an $\mathrm{EV}$ is parked, the decision to plug it in for charging, or not, is made taking into account its driver behavior and current SOC.

\section{Output Data}

The methodology described in sections IV A and IV B allows obtaining, for the period of one week, the following data: the periods during which EV are plugged-in and available to charge, the network bus to which EV are pluggedin, the EV power absorbed in each 30 min interval, the EV battery SOC evolution and the EV travelled distances.

\section{CASE Study}

Figure 1 shows the single line diagram of the MV grid from a rural area $(15 \mathrm{kV})$ used as test case in this research. It is composed by residential, industrial and commercial areas, thus allowing tracking each EV while commuting to and from work and to and from leisure activities. The power factor assumed for the conventional load is 0.96, whereas the specified voltage in the feeding point is 1.05 p.u.. There is a total of 7035 conventional cars enclosed in the geographical area covered by this grid and it was assumed that only one fast charging station exists, located in a robust area of the grid (bus 231), not prone to technical limits violations. The network has 309 buses, from which 115 have loads connected. The peak load is 7.3 MW (without EV consumption) and the energy consumption during a typical week is $789 \mathrm{MWh}$.

In order to perform the simulations, a typical weekly load diagram for this network was used. This diagram (dark grey area of Figure 4) was obtained by aggregating the load diagrams of the different types of consumers within the network. Regarding the studies performed, three simulations were run, considering: all EV as smart charging adherents (to evaluate the performance of the approach developed for the EV charging management performed by the aggregators) and all $\mathrm{EV}$ as dumb charging and multiple tariff $\mathrm{f}^{4}$ adherents (to evaluate the performance of the approach developed for the EV charging management performed by the DSO). For these studies, an EV integration level of $25 \%$ was considered (meaning that $1759 \mathrm{EV}$ were considered to exist in this area). In each simulation performed two situations are evaluated: the presence and absence of the grid monitoring performed by the DSO. While in the former the DSO might reduce the EV load to avoid the violation of the grid components' technical limits, in the latter it is assumed that the DSO never interferes with the EV charging. These two situations were evaluated for comparison purposes, with the objective of analyzing the influence that the DSO might have over the EV charging.

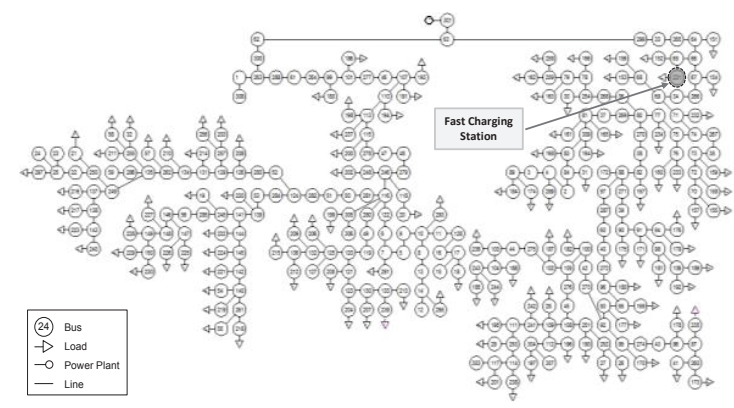

Figure 1. Single line diagram of the network.

\section{RESULTS}

\section{A. Mobility Patterns and EV Availability}

The journeys distribution during a week and a weekend day (Friday and Saturday) for the dumb charging, multiple tariff and aggregators' smart charging are presented in Figure 2. The curves for the three charging strategies follow the same trend. This is, in fact, an expected result, as the same assumptions were used to simulate the EV movement in all the scenarios addressed (the discrete-time, discrete-state Markov chain described in section IV B). During the week day (Friday), three peaks are clearly noticeable in the figure, two most likely related with household - work commuting (around $8 \mathrm{~h}$ and $18 \mathrm{~h}$ ), and the third, slightly after noon, probably related with people leaving their working places to have lunch somewhere else. In the weekend day (Saturday), probably due to the absence of the household - work commuting, the journeys are more distributed during the day.

In order to provide some insights about the places where the EV stay parked during the day, the number of EV parked

\footnotetext{
${ }^{4}$ The lower electricity price period assumed was that of the dual tariff policy currently implemented in Portugal: $22 \mathrm{~h}$ to $8 \mathrm{~h}$. More information can be found in: http://www.edpsu.pt/pt/particulares/tarifasehorarios/ (in Portuguese).
} 
in residential, commercial and industrial areas is presented in Figure 3. In what regards residential areas, as expected, there is a large number of EV parked during the night period, both on the week and on the weekend day. During the day, the number of EV parked in these areas is considerably lower during the week than during the weekend, probably due to the fact of most of the people not working during the weekend. The results are quite different for commercial and industrial areas, where the number of EV parked reaches the highest values during the day, both on week and weekend day. Still, while the number of EV parked in commercial areas reaches almost the same maximum value during all the days of the week, the number of EV parked in industrial areas is considerably lower during the weekend than during the week.

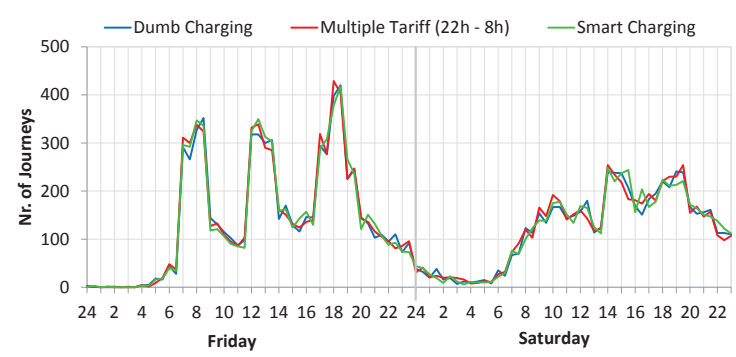

Figure 2. Journeys distribution during a week and a weekend day.

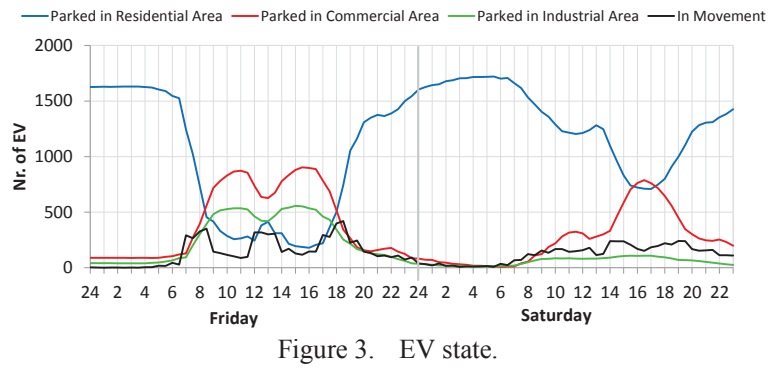

\section{B. Changes in Load Diagrams}

Figures 4, 5 and 6 show the load diagrams changes for the scenarios simulated, assuming an EV integration of $25 \%$.

As shown in Figure 4, with the dumb charging, the EV tend to charge mostly at the end of the day. The amount of power requested by the EV provokes a very large increase in the peak load, leading to the violation of the technical limits of several grid components. In order to avoid these violations, the DSO would have to override the aggregators' control signals and reduce $25.7 \mathrm{MWh}$ of the energy demanded by EV during the week (black areas in Figure 4). The load reduction is calculated with the heuristic described in section III $\mathrm{B}^{5}$.

Figure 5 shows the changes in the load diagram for the multiple tariff. The EV only charge at level 1 (slow charging) between $22 \mathrm{~h}$ and $8 \mathrm{~h}$, which is the period of time when the energy prices are lower. For this reason, there is a high number of EV connecting to the grid for charging at $22 \mathrm{~h}$ and the amount of power requested provokes the violation of the technical limits of some grid components. In order to avoid these violations, the DSO would have to reduce $21.1 \mathrm{MWh}$ of the energy demanded by EV during the week (black areas in

\footnotetext{
${ }^{5}$ This heuristic was coded in Python programming language, whereas the power flows were run in the $P S S / E$ software.
}

Figure 5). Again, this value was obtained using the heuristic described in section III B. The remaining EV load that appears outside the period $22 \mathrm{~h}-8 \mathrm{~h}$, is due to EV charging at level 2 (commercial areas) and 3 (fast charging station).

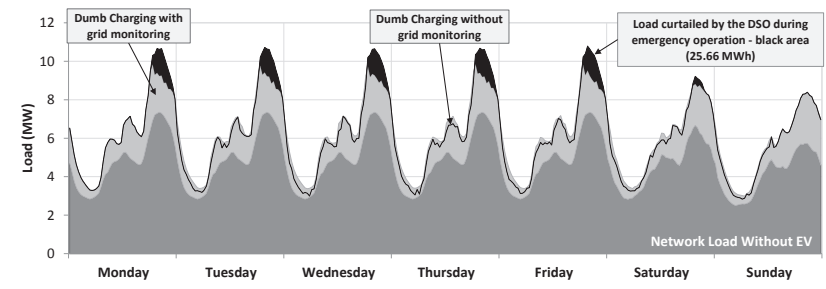

Figure 4. Load diagram in the dumb charging scenario.

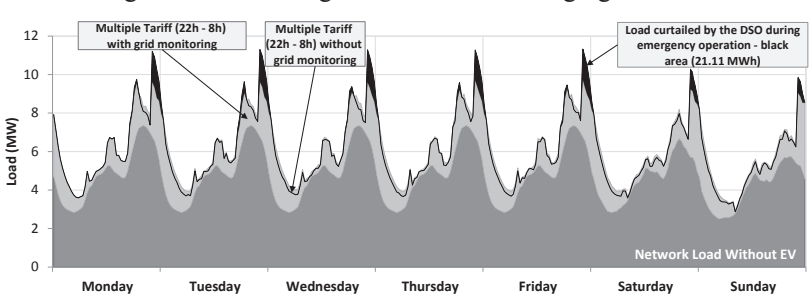

Figure 5. Load diagram in the multiple tariff $(22 \mathrm{~h}-8 \mathrm{~h})$ scenario.

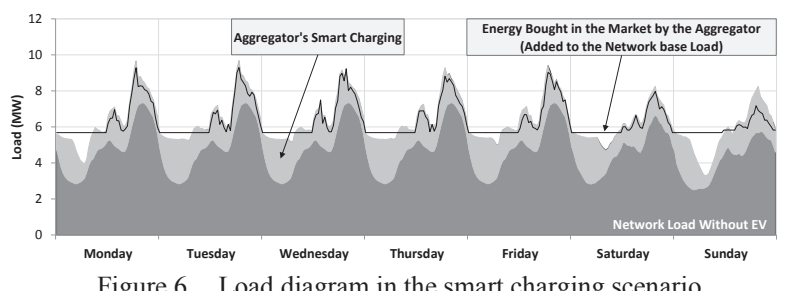

In what regards the smart charging, it was assumed the existence of aggregators, which are responsible for the EV charging management in normal operating conditions. Seeking to maximize their profit, the aggregators will try to buy energy in the periods when its price is lower and manage the "flexible EV" charging accordingly. For illustration purposes, it was assumed that the energy bought by the aggregators corresponds to the "valley filling" curve represented by the black line in Figure 6. In the following day, if deviations between the energy bought ("valley filling" curve) and the energy consumed by EV are registered, the aggregators will be penalized in the imbalance settlement. Thus, the maximization of the aggregators profit cannot disregard the minimization of the referred deviations. The light grey area in Figure 6, referred to as aggregators' smart charging, shows the results obtained with the "flexible EV" charging management performed by the aggregators. The "flexible EV" charging management was performed according to the optimization problem described in section III $\mathrm{A}^{6}$. As no technical violations were detected in the smart charging scenario, no EV load reduction was requested by the DSO, meaning that the DSO does not interfere with the aggregators' profit in this case.

\section{Deviations from the Energy Bought by the Aggregators}

The deviations between the energy bought in the markets by the aggregators (black line) and the energy consumed by

${ }^{6}$ This linear optimization problem was solved using the simplex method available in the LINGO 13.0 software. More information can be found in: http://www.lindo.com/index.php?option=com_content\&view=article\&id=2\&Itemid=10 
the EV (blue line), in the aggregators' smart charging, are shown in Figure 7 (red line). When the energy bought by the aggregators is higher than the EV consumption, it means that no further "flexible EV" are available for charging and thus the aggregator will have an energy surplus that should be sold in the intraday market. Conversely, when the EV consumption is higher than the energy bought, it means that the availability restrictions imposed by some of the "flexible EV" exhausted the possibility of the aggregator postpone further their charging. Thus they will start charging immediately. In these situations the aggregator will have an energy deficit that can be compensated by buying extra energy in the intraday market. It should be noted that these deviations would probably be greatly reduced if adequate forecasting techniques were used to determine the "flexible EV" availability.

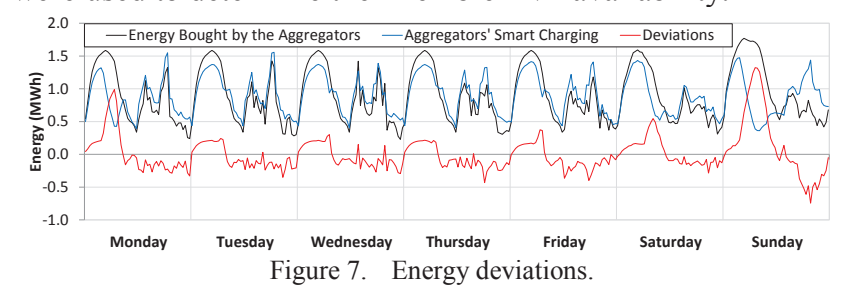

\section{Voltage Profiles}

In order to assess the worst voltage conditions that an EV integration level of $25 \%$ might lead to, the highest peak load registered during the simulations performed for each scenario was analyzed, and the corresponding voltage values were plotted in Figure 8. The extra power demanded by EV provokes a significant voltage drop along the grid, namely during the periods when the demand is higher, that, as Figure 8 shows, violate by far the lower limit of 0.90 p.u. in the dumb charging and multiple tariff $(22 \mathrm{~h}-8 \mathrm{~h})$ scenarios. These are the violations that trigger the emergency operating state and that obliges the DSO to curtail some of the EV load. After running the DSO grid management algorithm and reducing the EV load required, the voltages obtained were restored to normal values. The voltage drop is greatly reduced with the smart charging, where no violations were detected. The voltage profile for the peak load in the scenario without EV is also presented in Figure 8, for comparison purposes, as well as the reference voltage level stipulated by EN 50160 [15].

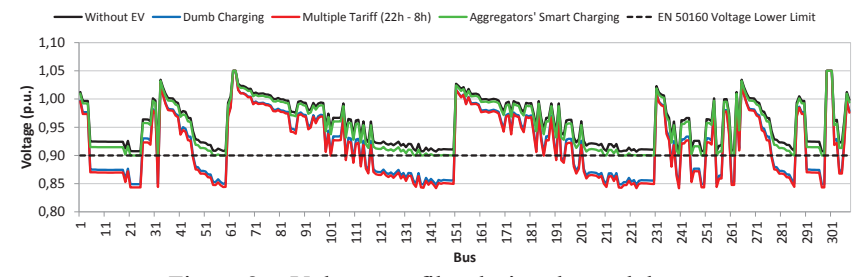

Figure 8. Voltage profiles during the peak hour.

\section{CONCLUSION}

The approach proposed in this work for the aggregator proved to be an efficient method to minimize the deviation between the energy bought in the market and the energy consumed by EV. Even so, some deviations were recorded in the case study analyzed, which would oblige the aggregator buying or selling the extra energy in the intraday market in order to avoid high penalizations in the imbalance settlement. Nonetheless, these deviations would probably be greatly reduced if adequate forecasting techniques were used to determine the "flexible EV" availability. The approach proposed for the DSO also proved to be very efficient, since it allowed performing the grid monitoring and managing the EV charging in order to solve all the voltage problems detected.

Both methodologies are suitable for quasi-real-time applications since they are capable of defining optimal (in the case of the aggregator management presented in section III A) or near-optimal (in the case of the DSO management presented in section III B) EV charging schedules in a very short period of time. The time needed to run the algorithms in a 3.16 GHz Intel Core 2 Duo CPU with 4.00 GB RAM, for the current case (grid with 309 buses and $1759 \mathrm{EV}$ ), was always lower than one minute for the 336 time steps analyzed.

For the DSO, the algorithm presented in section III B can be used as a tool to: detect the grid components that are subject to the more demanding operating conditions and that might need to be upgraded; monitor the grid and evaluate its operating conditions; and manage EV charging in quasi-realtime to mitigate voltage or line overloading problems. The algorithm of section III A can also be very helpful for aggregators, as it allows: defining the optimal bids for the dayahead markets; and managing EV charging in quasi-real-time with the purpose of minimizing the deviations between the energy bought in the market and the energy consumed by EV.

\section{REFERENCES}

[1] G. T. Heydt, "The Impact of Electric Vehicle Deployment on Load Management Straregies," Power Apparatus and Systems, IEEE Transactions on, vol. PAS-102, pp. 1253-1259, 1983.

[2] K. Schneider, et al., "Impact assessment of plug-in hybrid vehicles on pacific northwest distribution systems," in Power and Energy Society General Meeting - Conversion and Delivery of Electrical Energy in the 21st Century, 2008 IEEE, 2008, pp. 1-6.

[3] J. A. P. Lopes, et al., "Identifying management procedures to deal with connection of Electric Vehicles in the grid," in PowerTech 2009, pp. 1-8.

[4] K. Clement-Nyns, et al., "The Impact of Charging Plug-In Hybrid Electric Vehicles on a Residential Distribution Grid," Power Systems, IEEE Transactions on, vol. 25, pp. 371-380, 2010.

[5] J. A. P. Lopes, et al., "Integration of Electric Vehicles in the Electric Power System," Proceedings of the IEEE, vol. 99, pp. 168-183, 2011.

[6] E. Sortomme, et al., "Coordinated Charging of Plug-In Hybrid Electric Vehicles to Minimize Distribution System Losses," Smart Grid, IEEE Transactions on, vol. 2, pp. 198-205, 2011.

[7] O. Sundstrom and C. Binding, "Flexible Charging Optimization for Electric Vehicles Considering Distribution Grid Constraints," Smart Grid, IEEE Transactions on, vol. 3, pp. 26-37, 2012.

[8] M. D. Galus and G. Andersson, "Integration of Plug-In Hybrid Electric Vehicles into energy networks," in PowerTech, 2009 IEEE Bucharest, 2009, pp. 1-8.

[9] N. O'Connell, et al., "Electric Vehicle (EV) charging management with dynamic distribution system tariff," in Innovative Smart Grid Technologies (ISGT Europe), 2011 2nd IEEE PES International Conference and Exhibition on.

[10] S. Bending, et al., "Specification for an Enabling Smart Technology," Deliverable D1.1 of the European Project MERGE, August 2010.

[11] Z. Wei, et al., "Probabilistic wind power penetration of power system using nonlinear predictor-corrector primal-dual interior-point method," in Electric Utility Deregulation and Restructuring and Power Technologies, 2008. DRPT 2008. Third International Conference on, 2008.

[12] A. Papalexopoulos, "Challenges To On-line Opf Implementation," Power Systems, IEEE Transactions on, vol. 12, pp. 449-451, 1997.

[13] "Inquérito à mobilidade da população residente," INE - Instituto Nacional de Estatística, 2000 (in Portuguese).

[14] F. J. Soares, et al., "A Stochastic Model to Simulate Electric Vehicles Motion and Quantify the Energy Required from the Grid," PSCC, Sweden, 2011.

[15] "EN 50160 - Voltage characteristics of electricity supplied by public distribution systems", CENELEC, 2007. 